\title{
Discussions About the Feasibility of Using the Pediatric Physiological and Anatomical Triage Score in Earthquake Pediatric Patients
}

\author{
Run Lin, MB; ${ }^{1}$ Enjiang Lai, MB; ${ }^{1} \odot \mathrm{Hai} \mathrm{Hu}, \mathrm{MD}, \mathrm{PhD}^{2,3,4} \odot$
}

1. West China School of Medicine, Sichuan University, Chengdu, China

2. Emergency Office of West China Hospital, Sichuan University, Chengdu, China

3. China International Emergency Medical Team (Sichuan), Chengdu, China

4. Chinese National Comprehensive Emergency Medical Rescue Base, Chengdu, China

\author{
Correspondence: \\ Hai $\mathrm{Hu}, \mathrm{MD}, \mathrm{PhD}$ \\ No.37 Guoxue Alley \\ Wuhou District, Chengdu City, \\ Sichuan Province, PR China \\ E-mail: 412643356@qq.com
}

Conflicts of interest/disclaimer: The views expressed in the submitted article are the authors' and not an official position of the institution or funder. No conflicts of interest to declare.

\author{
Abbreviations: \\ AUC: Area Under Curve \\ BP: blood pressure \\ HR: heart rate \\ ICU: intensive care unit \\ PPATS: Pediatric Physiological and Anatomical \\ Triage Score \\ ROC: Receiver Operating Characteristic \\ TRTS: Triage Revised Trauma Score
}

Received: August 10, 2019

Accepted: October 06, 2019

doi:10.1017/S1049023X20000205

(C) World Association for Disaster and

Emergency Medicine 2020.
Lin R, Lai E, Hu H. Discussions about the feasibility of using the Pediatric Physiological and Anatomical Triage Score in earthquake pediatric patients. Prehosp Disaster Med. 2020;35(2):231-232.

To the Editor,

The article by Takashi Muguruma, et al is inspiring..$^{1}$ It compared a triage system designed for pediatric patients, Pediatric Physiological and Anatomical Triage Score (PPATS), with a widely used triage method for generic patients, Triage Revised Trauma Score (TRTS), ${ }^{2}$ and suggested that PPATS is a viable and useful triage method specifically for pediatric patients. $^{1}$

However, different disasters have their own characteristics of casualties. ${ }^{3}$ The pediatric patients in this article were from the Japan Trauma Data Bank, and they may be different from the patients in earthquake. Previous studies have compared the effectiveness of different triage methods on earthquake trauma patients, ${ }^{4}$ but few studies have focused on triage methods for earthquake pediatric patients. To verify the effectiveness of this method in earthquake pediatric patients, we repeated this study with patients from the Earthquake Victim Database.

We conducted a retrospective research comparing PPATS with TRTS and collected the information of all injured patients whose age was no greater than 15 years old in the Earthquake Victim Database. To calculate the triage scores, we gathered the patients' age, respiratory rate $(\mathrm{RR})$, heart rate $(\mathrm{HR})$, systolic blood pressure $(\mathrm{BP})$, and Glasgow Coma Score (GCS) upon admission, as well as the usage of live-saving interventions; we also used the patient's diagnosis on admission to determine whether or not the patient had anatomical abnormality. Based on this, we calculated the PPATS score and TRTS score for each patient. Whether the patient was admitted to the intensive care unit (ICU) was set as the outcome, and that information was also collected. Microsoft Excel 2019 (Microsoft Corp.; Redmond, Washington USA) was used to gather and filter information and also calculate the triage scores. To evaluate the two triage methods, we used MedCalc 18.11.3 (MedCalc Software; Ostend, Belgium) to perform a Receiver Operating Characteristic curve (ROC curve) analysis to determine their predictive ability and performed an Area Under Curve (AUC) comparison between the two methods.

Excluding instances that lack of basic information $(n=4)$, admission to ICU information $(n=53)$, and information crucial to calculate triage scores $(n=408)$, a total of 1,585 cases were enrolled in the study. The mean age was $11.23(\mathrm{SD}=3.06)$ years with 752 male patients (47.44\%). The TRTS scored ranged from eight to 12 and PPATS scored raged from zero to 11. The ROC curve of the two triage methods are shown in Figure 1. The AUC of PPATS and TRTS are 0.704 and 0.560, respectively; the AUC of PPATS is significantly greater than that of TRTS $(\mathrm{P}<.001$; Table 1$)$.

The result shows that PPATS is a relatively effective triage method for pediatric patients compared with TRTS; we approve the original article's conclusion that PPATS is superior to TRTS. What's more, the result shows the effectiveness of PPATS in an earthquake. However, our study is also limited by its retrospective design and missing data; a large-scale, multi-center, prospective study is needed to better evaluate its effectiveness and capability.

One of the main advantages of PPATS is that it not only differs pediatric patients and adult patients, but it utilizes a percentage value according to the patient's age rather than a fixed value when evaluating BP and HR scores. The score shows the severity of the 


\begin{tabular}{|l|c|c|c|c|c|}
\hline & AUC & SE & 95\% Cl & $\begin{array}{c}\text { Difference Between } \\
\text { Areas }\end{array}$ & P Value \\
\hline PPATS & 0.704 & 0.0373 & $0.681-0.726$ & 0.144 & $<.001$ \\
\hline TRTS & 0.560 & 0.0274 & $0.535-0.585$ & & \\
\hline
\end{tabular}

Table 1. AUC Comparison between PPATS and TRTS

Lin (๑) 2020 Prehospital and Disaster Medicine

Abbreviations: AUC, Area Under Curve; PPATS, Pediatric Physiological and Anatomical Triage Score; SE, Standard Error; TRTS, Triage Revised Trauma Score.

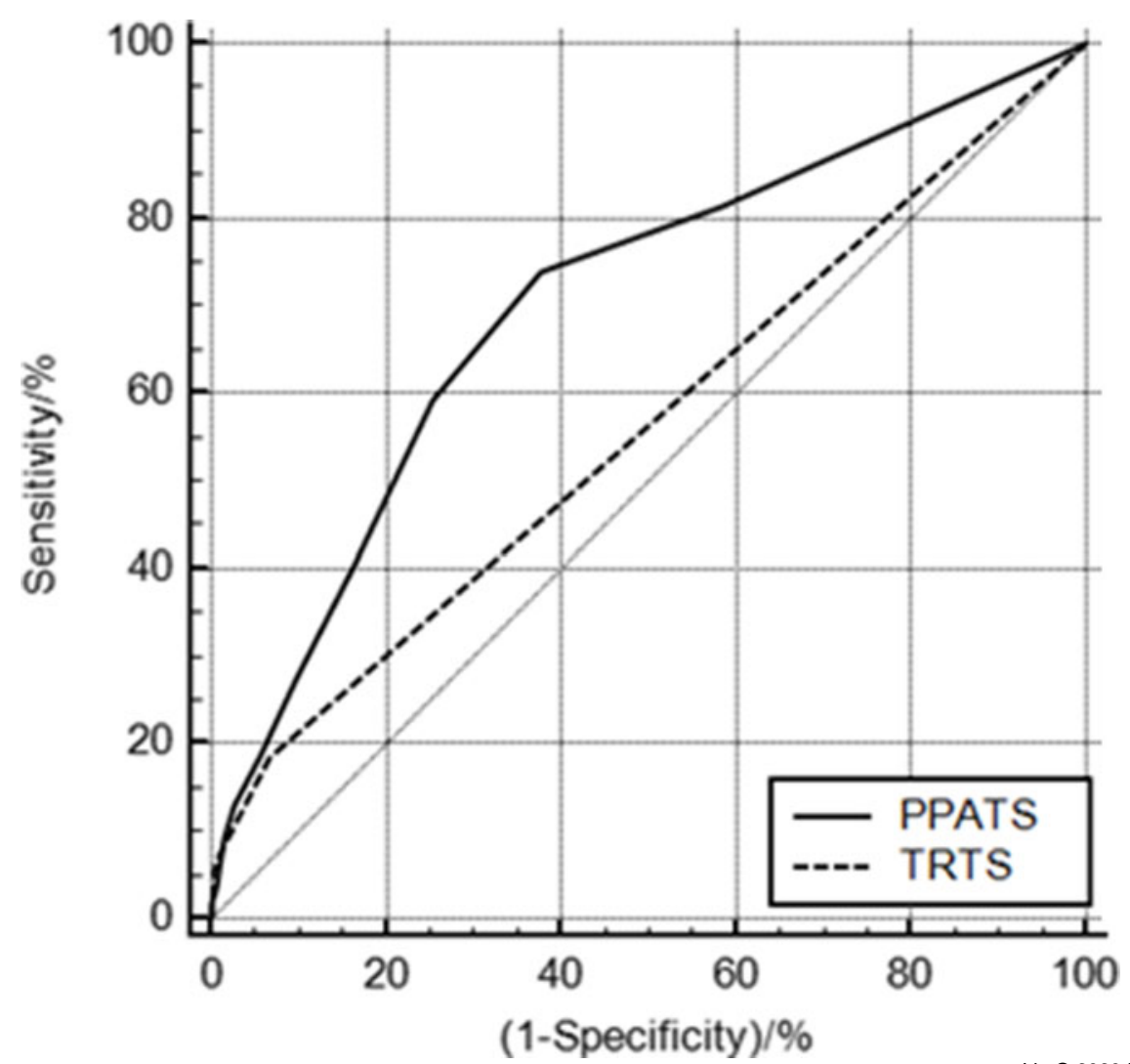

Figure 1. The ROC Curves of PPATS and TRTS.

Lin (๐) 2020 Prehospital and Disaster Medicine

Abbreviations: PPATS, Pediatric Physiological and Anatomical Triage Score; ROC, Receiver Operating Characteristics; TRTS, Triage Revised Trauma Score.

patients within the same age better, thus minimizing the deviation caused by age on these values when triaging. However, in a disaster setting such as an earthquake, hurricane, and so on, where the number of injured massively overwhelm the number of health care providers and an electronic triaging system is not accessible, PPATS might be too complicated to carry out as the BP and HR standard is different with each age. ${ }^{5} \mathrm{~A}$ simplified standard for BP and HR evaluation might be a good alternative under these circumstances.

In conclusion, PPATS is an effective method to triage pediatric patients in an earthquake, and may be adopted in the triaging of pediatric patients.

\section{References}

1. Toida C, Muguruma T, Takeuchi I, Morimura N. Validation of the Pediatric Physiological and Anatomical Triage Score in pediatric patients with burn injuries. Prehosp Disaster Med. 2019;34(Suppl 1):s61.

2. Toida C, Muguruma T, Abe T, et al. Introduction of Pediatric Physiological and Anatomical Triage Score in mass-casualty incident. Prehosp Disaster Med. 2018;33(2):147-152.
3. Zhang N, Huang H. Assessment of world disaster severity processed by Gaussian blur based on large historical data: casualties as an evaluating indicator. Nat Hazards. 2018:92:173-187.

4. Hu H. Comparison of six disaster triage methods using the Wenchuan Earthquake Victim Database. Prehosp Disaster Med. 2017;32(Suppl 1):s234.

5. Koziel JR, Meckler G, Brown L, et al. Barriers to pediatric disaster triage: a qualitative investigation. Prehosp Emerg Care. 2015;19(2):279-286. 